\title{
Zur Frage der Erhöhung des Brenztraubensäure-Blutspiegels bei multipler Sklerose
}

\author{
Von \\ William Garitano \\ Aus dell Forsclungslaboratorium der Neurologischen Universitätsklinik Basel (Direktor: Prof. Dr. F. Georgi)
}

(Der Schriftleitung zugegangen am 10. Januar 1964)

\begin{abstract}
In Anbetracht der widersprüchlichen Literaturergebnisse wurde an 37 Personen (20 MS und 17 Kontrollen) der Brenztraubensäure-(„BTS“)-gehalt im Nüchternblut und nach Belastung mit Glucose mit neuen, spezifischeren Methoden nochmals bestimmt. Die Bestimmung erfolgte einerseits als Dinitrophenylhydrazon andererseits enzymatisch mit der „Test-Kombination Boehringer". Die beiden Methoden ergaben annähernd übereinstimmende Werte.

Es konnte eine statistisch gesicherte, pathologische Erhöhung der BTS sowohl im Nüchternblut wie auch nach Belastung festgestellt werden. Die „Normalwerte“ schwanken zwischen 0,3-0,7 mg\% mit einem Mittel von 0,47 mg\%. Demgegenüber weist die MS-Gruppe einen Mittelwert von $0,76 \mathrm{mg} \%$ auf; hohe Werte fanden sich vor allem bei Frauen. Ein ursächlicher Zusammenhang zwischen BTS-Spiegel und MS scheint kaum wahrscheinlich; die pathologische Erhöhung dürfte vielmehr die Folge von Leber- oder Nierenschädigungen, motorischer Unruhe, emotioneller Erregung usw. sein.
\end{abstract}

\begin{abstract}
To clarify contradictory findings in the literature, the pyruvic acid level of the blood was determined in 37 fasting patients (20 MS and 17 controls), using newer and more specific methods. The procedure was repeated two hours after the administration of glucose. The determinations of the pyruvic acid level were made using dinitrophenylhydrazone and partially with the enzymatic method. The values obtained were found to be similar for both methods. A definite pathologically increased level of pyruvic acid could be established both in fasting blood and after loading with glucose. The „normal“ values varied between $0.3-0.7 \mathrm{mg} \%$ with an average fasting value of $0.47 \mathrm{mg} \%$ $(0.50 \mathrm{mg} \%$ after loading with glucose), whereas the MS group showed an average value of $0.76 \mathrm{mg} \%$ (fasting) and $0.92 \mathrm{mg} \%$ (after loading with glucose). High values were found chiefly in female patients. A causal relationship between the raised pyruvic acid level and multiple sclerosis appears improbable. The pathological increase is more likely ascribable to liver and kidney damage, physical and emotional tension, etc.
\end{abstract}

Die Frage der Erhöhung des Brenztraubensäure(„BTS")-Spiegels bei multipler Sklerose („MS“) wurde von JONES, JONES und BUNCH (1) im Jahre 1950 aufgeworfen und bejaht, nachdem sich schon vorher einige Autoren (Wortis und Bueding (2), Markees (3) sowie ENDTZ und SCHuller (4)) mit deren Verhalten bei verschiedenen anderen Erkrankungen befaßt hatten. Die weitere Forschung auf diesem Gebiet hat zu stark divergierenden Ansichten geführt. So glauben u. a. ERvenich (5), SERCL und Johnova (6) und auch Gros (7) bei einem bis zwei Dritteln der MS-Fälle das Vorliegen erhöhter BTS-Werte bestätigen zu können. Demgegenüber sind jedoch Georgi und Beutitien (8), Markees (9) sowie Bauer (10) der Meinung, daß eine artifizielle Erhöhung der BTS-Werte im Nüchtern-Blut nur vereinzelt und zwar, als Folge körperlicher Unruhe oder infolge nịcht kunstgerechter Blutentnahme gefunden werde.

Eine ähnliche Unsicherheit der Interpretation herrscht bei den Untersuchungen mittels. Belastung durch größere Mengen Glucose (20-100 g oral) und Verfolgung des BTS-Spiegels innerhalb der nächsten 1-3 Stunden. Jeanes und Cumings (11), McArdie (12) sowie Sercl und Johnova (6) stellen nach Belastung eine deutliche Erhöhung des BTS-Spiegels im Blut von MS-Patienten fest, während HENNEMAN und Mitarbeiter (13) unter den gleichen Bedingungen vor allem ein abnormes Verhalten der Citronensäure gesehen haben; die dabei gleichzeitig auftretende leichte Anhäufung von BTS und Milchsäure wird von RIEbeling (14) ebenso wie die Angaben für die $\alpha$-Ketoglutarsäure noch der Norm zugerechnet. MAR KEES und Mitarbeiter (9) finden jedoch die $\alpha$-Ketoglutarsäure-Werte schon im Nüchtern-Blut in über zwei Dritteln der MS-Fälle pathologisch erhöht. $\mathrm{Zu}$ einer ähnlichen Feststellung bezüglich $\alpha$-Ketoglutarsäure gelangt auch MCARDLE (12), während CENDROwSKI $(15,16)$ wohl den BTS-, nicht aber den Milchsäure-Spiegel erhöht findet.

Bei der zentralen Stellung, welche die BTS zwischen der Glykolyse und dem Krebszyklus einnimmt, ist es natürlich nicht verwunderlich, daß einige ihrer Umund Abbauprodukte ebenfalls gestörte Verhältnisse aufweisen. Außerdem findet z. B. Juverh (17) den Zuckerabbau bei MS und unter verschiedenen anderen Bedingungen schon auf der Höhe der Aldolase verändert, führt dies jedoch eher auf erhöhten Energieverbrauch infolge Muskelspannung, Tremor, Unruhe usw. zurück. Andererseits ist bei der Diversität der bisher mitgeteilten Ergebnisse zu berücksichtigen, daß die meisten Untersuchungen mit relativ unspezifischen chemischen Methoden durchgeführt wurden, welche im allgemeinen auf die Gesamtheit der Ketosäuren ansprechen, von denen 
allerdings die BTS den Hauptanteil ausmacht. Das am häufigsten angewandte Verfahren zur BTS-Bestimmung beruht auf der Bildung des 2,4-Dinitrophenylhydrazons der BTS in saurer Lösung. Der entstehende rote Farbkomplex läßt sich dann kolorimetrisch bestimmen. $\mathrm{Da}$ aber, wie gesagt, alle Ketosäuren die Fähigkeit zur Hydrazonbildung besitzen, werden viele unerwünschte Substanzen im Serum miterfaßt, und die resultierenden BTS-Werte liegen daher im allgemeinen zu hoch. Im Bestreben, die Spezifität der Erfassung zu steigern, gelangten schließlich einige methodische Modifikationen zur Anwendung. Es ist daher wenig verwunderlich, $\mathrm{da} ß$ von verschiedenen Autoren stark voneinander abweichende Normalwerte angegeben werden (z. B. Gastaldi (19) 0,43, Geigy (20) 1,1 als Mittelwert!), ein Umstand, der die Festlegung verbindlicher Normgrenzen erheblich erschwert und die Vergleichbarkeit einzelner Publikationen u. U. überhaupt in Frage stellt.

Erst die neuerdings eingeführten enzymatischen Methoden erlauben mit Sicherheit, die BTS spezifisch zu erfassen. Wir haben uns daher, und weil die bisherigen Literaturangaben an den erwähnten Widersprüchen leiden, die Aufgabe gestellt, das Problem erhöhter BTS-Werte bei MS im Nüchtern-Blut und nach Belastung mit Glucose erneut zu überprüfen, wobei wir uns zweier spezifischer Methoden bedienten, die bisher nur in wenigen Untersuchungen angewendet wurden.

\section{Methodik}

An 37 Patienten wurden insgesamt 72 Blutuntersuchungen durchgeführt. Und zwar wurden in einer ersten Serie 11 MS-Patienten und 10 Kontrollfälle (keine zentraldestruktiven Fälle, vorwiegend Diskusaffektionen) in der von MarkeEs (3) modifizierten Methode nach FrIedemanN-HaUgen untersucht, wobei allerdings der letzte Schritt in einer zusätzlichen Modifikation (Ausschüttlung mit Xylol) nach den Angaben von Salvatore und BocchinI (18) besteht; es wird damit eine annähernd spezifische Erfassung der BTS erzielt. In einer zweiten, späteren Versuchsserie wurden weitere 9 MSPatienten und 7 Kontrollfälle mittels der enzymatischen Methode der Fa. C. F. Boehringer, Mannheim, geprüft. Zur Bestimmung des Nüchternwertes wurde dem liegenden Patienten Blut aus der ungestauten Vene entnommen; danach wurden $50 \mathrm{~g}$ Glucose in einem Glas Wasser verabreicht. Zwei Stunden später erfolgte eine zweite Blutentnahme, die nach der jeweils gleichen Methode untersucht wurde.

\section{Chemische Methode}

$3 \mathrm{~m} l$ Vollblut werden sofort mit der 5-fachen Menge Trichloressigsäure enteiweißt und zentrifugiert. Der klare Überstand wird $5 \mathrm{Min}$. im Wasserbad gekocht. $3 \mathrm{~m} l$ davon werden mit $1 \mathrm{~m} l 0,1 \%$ 2,4-Dinitrophenylhydrazin in $2,5-n \mathrm{HCl}$ versetzt. Nach 5 Min. Reaktionszeit bei $37^{\circ}$ wird mit $2 \mathrm{~m} l$ Xylol kräftig geschüttelt und dann $10 \mathrm{Min}$. bei $3000 \mathrm{U} / \mathrm{Min}$. zentrifugiert. $1 \mathrm{ml}$ Xylolschicht wird sodann mit $6 \mathrm{ml}$ alkoholischer $\mathrm{KOH}(2,5 \%$ in $95 \%$ Athanol) versetzt. Nach weiteren $5 \mathrm{Min}$. mißt man die Extinktion im Spektrophotometer bei Wellenlänge $490 \mathrm{~m} \mu$.

\section{Enzy'matische Methode}

Die Bestimmung erfolgt mit $4 \mathrm{~m} / \mathrm{Blut}$ aus ungestauter Vene, wobei sofort mit eiskalter Perchlorsäure enteiweißt und nach der Vorschrift der Fa. Boehringer weiterbehandelt wird. Das Meßprinzip beruht auf der Oxydation von reduziertem Phosphorpyridinnucleotid (DPN-H) und Ablesung der Extinktion bei Wellenlängc $345 \mathrm{~m} \mu$ vor und nach Zugabe von Lactatdehydrogenase. Aus der Extinktionsdifferenz wird der Brenztraubensäuregehalt berechnet.

\section{Ergebnisse}

Bei einem Vergleich der chemischen mit der enzymatischen Methode stellen wir vorerst fest, daß die Nüchternwerte der beiden Kontroll-Kollektive fast identische Mittelwerte und Streuungen aufweisen, ein Beleg dafür, daß die Xylol-Modifikation von Salvatore und BocchInI (18) eine hohe Spezifität für BTS aufweist. Auch unter Belastung mit Glucose, wonach mit einem beträchtlichen Anfall anderer Ketokörper zu rechnen ist, zeigt das mit der Xylol-Methode bestimmte KontrollKollektiv nur etwa $10 \%$ höhere Durchschnittswerte als die enzymatisch erfaßte Probandengruppe. Wir halten daher die beiden Methoden für annähernd gleichwertig, und unsere weiteren Aussagen basieren auf der Vereinigung aller sich klinisch entsprechenden Versuchswerte.

Im allgemeinen liegen die so gefundenen Vergleichswerte von nicht zentralnervös, nicht muiskel- und nicht lebergeschädigten Kontrollfällen wesentlich tiefer als die beispielsweise von MARKEes (3) und anderen (4) genannten Mittelwerte für die Phenylhydrazonmethode von rund $0,8 \mathrm{mg} \%$. Mit durchschnittlich etwa $0,5 \mathrm{mg} \%$ (Streuung $0,3-0,65 \mathrm{mg} \%$ ) für Nüchternblut decken sie sich jedoch weitgehend mit den $0,53 \mathrm{mg} \%$ von BAUER (10) oder den $0,56 \mathrm{mg} \%$ von REDETzKI und Mitarbeitern (21), beide mit Hilfe des enzymatischen Testes ermittelt. Die Belastung mit Glucose hat beim Kòntrollkollektiv keinen namhaften Einfluß, indem 2 Stunden nach Belastung nur ein um knapp 10\% höherer Mittelwert erhalten wird.

Die MS-Patienten weisen nüchtern mit einem durchschnittlichen BTS-Spiegel von $0,76 \mathrm{mg} \%$ eine deutliche Erhöhung gegenüber den Kontrollen mit $0,47 \mathrm{mg} \%$ auf. Dieser erhöhte Mittelwert beruht nicht nur auf drei extrem pathologischen Werten, sondern auf einem generell höheren BTS-Gehalt im Blut. Während die Hauptmasse der Kontrollwerte im Bereich zwischen $0,4-0,56 \mathrm{mg} \%$ liegt, findet sich bei der MS-Gruppe die größte Häufigkeit im Bereich zwischen 0,56-0,75 $\mathrm{mg} \%$. Zudem ist auch die Streuung der Einzelwerte in der MS-Gruppe signifikant größer $(P=0,001)$ als diejenige des Kontrollkollektivs. - Über den Vergleich der beiden unter Glucosebelastung stehenden Gruppen läßt sich im Prinzip dasselbe aussagen, mit dem Unterschied allerdings, daß die Mittelwerte insgesamt, ausgeprägter jedoch bei MS, um einiges höher liegen $(0,92$ resp. $0,50 \mathrm{mg} \%$ ). Auch hier gilt die Feststellung einer signifikant größeren Streuung der MS-Gruppe $(P=$ $0,001)$. 
In übersichtlicher Darstellung sind diese Befunde in Abbildung 1 zusammengefaßt. Wir erkennen darin die Verschiebung der Schwerpunkte beim MS-Kollektiv im Vergleich zu den Kontrollen sowie die Tatsache, daß die MS-Patienten auf Glucosebelastung mit einer stärkeren Erhöhung der BTS-Werte ansprechen als die Kontrollen. Schließlich läßt die Darstellung offensichtlich werden, daß in statistischer Hinsicht die Häufigkeiten der untersuchten Wertgruppen nur im logarithmischen Maßstab annähernd ",normal" verteilt sind; Signifikanzberechnungen von Unterschieden waren deshalb auf logarithmischer Basis vorzunehmen.

Abbildung 2 gibt die Reaktion auf Glucosebelastung in detaillierter Form wieder. Es sind hier die absoluten Differenzen der Werte nach Belastung minus Nüchternwert aufgetragen. Allgemein läßt sich feststellen, daß die größten positiven Differenzen bei an sich schon hohem BTS-Nüchternspiegel auftreten. Deutlich gelangt auch die pathologisch verstärkte BTS-Erhöhung bei der MS-Gruppe zur Darstellung. In diesem $\mathrm{Zu}-$ sammenhang ist zu erwähnen, daß Frauen sowohl hinsichtlich Nüchternwert als auch hinsichtlich Reaktion auf Glucosebelastung stärker pathologische Werte aufweisen. Die kleinen Vergleichszahlen dieser Untersuchung erlauben jedoch keine sichere Aussage darüber, ob auch hier eine Geschlechtsdifferenz manifest sei, wie dies beispielsweise von RIEDER und WüTHRICH $(22,23)$ für das Gesamteiweiß des Liquors bei MS hat bewiesen werden können.

\section{Diskussion}

Die vorliegende Untersuchung hat ergeben, da $\beta$ die chemische Erfassung der BTS mit der Methode von Markees, modifiziert nach Salvatore und Bocchini, sowie die enzymatische Methode nach der Vorschrift der $\mathrm{Fa}$. Boehringer zu den gleichen Schlußfolgerungen führen und in guter Übereinstimmung "Normalwerte“ im Bereich von etwa $0,3 \mathrm{mg} \%$ bis $0,7 \mathrm{mg} \%$ BTS ergeben. Den Ausdruck „Normalwerte“ haben wir darum in Anführungszeichen gesetzt, weil es sich mehrheitlich nicht um Proben von Gesunden, sondern vorwiegend um solche von Patienten mit Erkrankungen streng umschriebener Lokalisation und meist mechanischer Genese (Discopathien) handelt. Da sich unsere Mittelwerte $(0,50 \mathrm{mg} \%)$ aber in guter Übereinstimmung befinden mit den Angaben von Bauer (10) $(0,53 \mathrm{mg} \%)$ und denjenigen von REDETZKI (21) $(0,56 \mathrm{mg} \%)$, welche ebenfalls mit spezifischen enzymatischen Methoden erhalten wurden, dürfen wir unser Kontrollkollektiv mit einiger Sicherheit als für Normale repräsentativ annehmen. Auf alle Fälle steht fest, daß der normale Bereich von BTS im Blut wesentlich tiefer anzusetzen ist als dies auf Grund der früheren chemischen Untersuchungen den Anschein hatte (vgl. auch (24)). Daß die BTS-Werte bei MS-Patienten im allgemeinen höher liegen als bei den Kontrollfällen, ist natürlich kein Indiz dafür, daß die primäre Ursache der MS in dieser Störung zu suchen ist. Die meisten MS-Patienten weisen relativ leicht erhöhte BTS-Werte auf, nur etwa ein Drittel der Fälle zeichnet sich durch einen extrem pathologischen Gehalt im Nüchtern-Blut aus. Nach
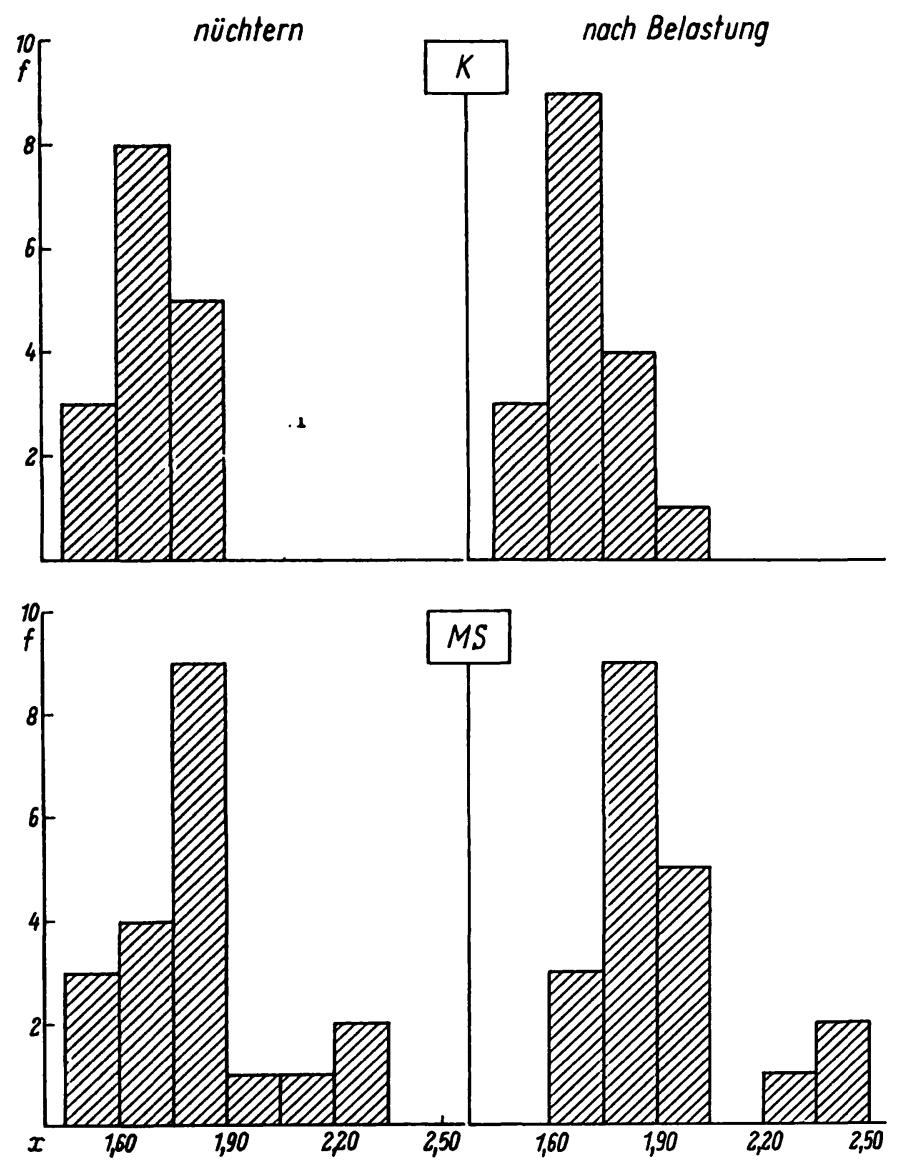

Abb. 1

Häufigkeitsverteilung der Brenztraubensäurewerte bei MS und Kontrollen; bei Blutentnahme nüchtern und nach Glucosebelastung $\mathrm{K}=$ Kontrollen; $\mathrm{MS}=$ Multiple Sklerosen; $\mathrm{f}=$ Frequenz der Werte bestimmter Größenklassen. Abszisse = Brenztraubensäurcwerte, ausgedrückt in $\mathrm{X}=\log \left(10^{2} \cdot \mathrm{mg} \%\right)$

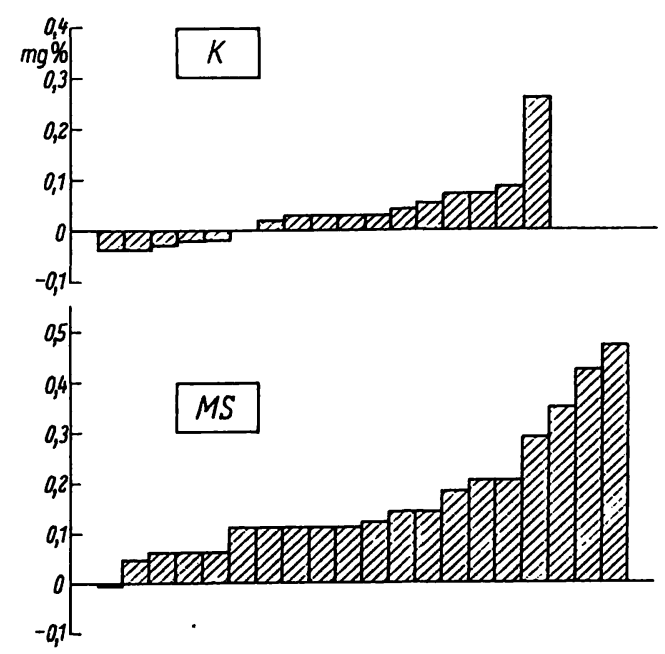

Abb. 2

Brenztraubensäuregehalt im Blut bei MS und Kontrollen; Differenz zwischen den Werten nach Glucosebelastung (B) und nüchtern (A). Ordinate: Differenz B-A in $\mathrm{mg} \%$; - Abszisse: Werte der 17 Kontrollen und $20 \mathrm{MS}$ in ansteigender Reihe 
eigenen klinischen Beobachtungen handelt es sich dabei aber nicht um die schwersten Fälle; hingegen scheinen vorwiegend Frauen an den höheren Werten beteiligt zu sein.

Erhöhte BTS-Spiegel lassen sich bei einer derartigen Vielzahl von Erkrankungen und Zuständen feststellen $(25,26), \mathrm{da} \beta$ es mehr als fraglich erscheint, ob diese Erhöhung überhaupt in irgendeiner ursächlichen Beziehung zur Multiplen Sklerose steht, finden wir doch pathologische Werte z. B. bei Leberparenchymschädigungen verschiedener Genese, bei Schizophrenie, Diabetes mellitus, chronischen Nierenleiden, Herzinsuffizienz, akuten Infektionen, malignen Tumoren, Hyperemesis gravidarum, Traumata am Zentralnervensystem usw. In diesem Zusammenhang ist ferner darauf hinzuweisen, daß nach den Befunden von Georgi und Beuthien (8) und Rieder (27) etwa bei der Hälfte der MS-Patienten mit Hilfe des kombinierten Hippursäuretests gestörte Leberfunktionen (häufig mit gleichzeitigem Nierenschaden) feststellbar waren. Die erhöhten BTSWerte könnten demnach eine bloße Folge dieser Organschädigungen sein. - Wenn somit eine klinische Bedeutung des untersuchten BTS-Gehaltes unwahrscheinlich erscheint, so sind doch einige statistisch interessante Befunde zu erheben. Beim Vergleich der beiden Kollektive MS/Kontrollen ergibt sich sowohl für das NüchternBlut wie für den 2-Stunden-Wert nach Belastung mit Glucose ein signifikant höherer Durchschnittswert für die MS-Gruppe. Letztere spricht auch auf Belastung eindeutig stärker an und zeichnet sich zudem durch eine gesichert breitere Streuung der Einzelwerte als die entsprechende Kontrollgruppe aus.

Unsere Schlußfolgerungen, zu denen wir auf Grund einer spezifisch verfeinerten Methodik gelangt sind, decken sich demnach mit den früheren Literaturangaben, die allerdings auf einem generell höheren Wertniveau bei MS-Patienten häufig eine pathologische Erhöhung des BTS-Spiegels im Blut feststellten. Andererseits müssen wir aber auch GeORgr und Mitarbeitern zustimmen, daß Werte über $1 \mathrm{mg} \%$ (nüchtern) oft durch arbeitstechnische Mängel oder durch die motorische Unruhe des Patienten bedingt sein können. Es muß ja auch berücksichtigt werden, daß der MS-Patient im Spital meist in der Lage eines chronisch Kranken ist, der gerade einen Rückschlag seiner zuvor mehr oder weniger stabilisierten Gesundheit erleidet. Die emotionelle Erregung und Angst, die in einer solchen Situation fast obligat ist, dürfte - sei sie auch noch so gedämpft nicht ohne Wirkung auf einen so empfindlichen Indikator wie der BTS-Spiegel sein. - Interessante Anknüpfungspunkte mag in diesem Zusammenhang die im Tierversuch erwiesene Beeinflußbarkeit des BTS-Blutspiegels durch Catecholamine (26) andeuten.

Herrn Prof. Dr. F. Georgr danke ich für die wertvolle Unterstützung und Kritik der vorliegenden Arbeit. Für praktische Ratschläge bei der Ausführung der Untersuchungen und für die Hilfe bei der statistischen Berechnung der Ergebnisse bin ich Herrn Dr. H. P. RIEDER zu großem Dank verpflichtet.

\section{Literatur}

1. Jones, H. H., H. H. Jones Jun. und L. D. Bunch, Ann. Int. Med. 33, 831 (1950). - 2. Wortis, H. und E. Bueding, Transact. Amer. Neurol. Ass. 66, 90 (1940). - 3. MarkeEs, S., Zschr. klin. Med. 145, 354 (1949); Helvet. med. acta 17, 537 (1950); Helvet. physiol. pharmacol. acta 9, 30 (1951); Experientia (Basel) 7,314 (1951); Schweiz. med. Wschr. 81, 1145 (1951); Dtsch. med. Wschr. 27/28, 971 (1953); Experientia (Basel) 11/5, 205 (1955). 4. Endtz, L. J. und E. Schuller, Presse méd. (Paris) 68, 1933 (1960). - 5. Ervenich, P., Aerztl. Praxis 4, 146 (1952); Aerztl. Forschg., Wörishofen 7, 55 (1953). - 6. SERCL, M. und B. JoHNova, Confinia neurol. 16, 177 (1956). - 7. Gros, H., E. J. KIRNBERGER und K. H. SchIFFER, Münch. med. Wschr. 97, 130 (1955). - 8. Georgi, F. und A. Beuthien, Confinia neurol. 15, 32 (1955). - 9. Markees, S., D. Jordan und F. Georgi, Verh. Dtsch. Ges. inn. Med., 61. Kongreß 1955, S. 374. - 10. Bauer, H., Biochem. Z. 327, 491 (1956). - 11. Jeanes, A. L. und J. N. Cumings, Confinia neurol. 18, 397 (1958). - 12. MCArdle, B., K. K. Mackenzie und G. R. Webster, J. Neurol. (London) Neurosurg. Psychiat. 23, 127 (1960). - 13. Henneman, D. H., M. D. Altschule, R. M. Goncz und L. Alexander, A. M. A. 。
Arch. Neurol. Psychiat. 72, 688 (1954). - 14. Rrebeling, C., Referat in Zbl. f. d. gesamte Neurol. und Psychiat. 132, 243 (1955). - 15. Cendrowskr, W., Odbitka Z. „Polskiego Tygodnika Lekarskiego" R. 15, No. 35 (1960). - 16. CENDrowskr, W. und K. Murawskr, Odbitka Z. „Polskiego Tygodnika Lekarskiego" R. 14, No. 15 (1959). - 17. JuvETH, L., Zschr. klin. Med. 154, 569 (1957). - 18. Salvatore, F. und V. Bocchini, Clin. chim. Acta (Amsterdam) 6, 109 (1961). 19. Gastaldr, A., Ann. ostetr. ginec. 77, 385 (1955). - 20. Dokumenta Geigy, Wissenschaftl. Tabellen (1960). — 21. RedETzKI, H., H. BloEdoRn und H. W. BANsi, Klin. Wschr. 34, 845 (1956). 22. Wüthrich, R., H. P. RIEDER und J. B. MeYer, Nervenarzt, Berlin 34, 32 (1963). - 23. RIEDER, H. P. und R. WÜTHRICH, Klin. Wschr. 40, 1070 (1962). - 24. Gloster, J. A. und P. Harris, Clin. chim. Acta (Amsterdam) 7, 206 (1962). - 25. Lasch, F., Dtsch. med. Wschr. 78, 975 und 985 (1953); Klin. Wschr. 31, 941 (1953); Acta neuroveget., Wien 9, 1 (1954). - 26. GEX, K. F. und A. Pletscher, Helvet. physiol. pharmacol. acta 19, C 70 (1961); Experientia (Basel) 17, 25 (1961). - 27. RIEDER, H. P., Zschr. klin. Med. 154, 87 (1956).
Dr. William Garitano, Forschungslaboratorium der Neurolog. Universitäts-Klinik, Basel (Schweiz), Socinstraße 55 